\title{
The Interacting Generalized Ricci Dark Energy Model in Non-Flat Universe
}

\author{
Masashi Suwa*, Koji Kobayashi, Hisashi Oshima \\ School of Pharmacy, Nihon University, Chiba, Japan \\ Email: suwa.masashi@nihon-u.ac.jp
}

Received 7 February 2015; accepted 25 February 2015; published 28 February 2015

Copyright (C) 2015 by authors and Scientific Research Publishing Inc.

This work is licensed under the Creative Commons Attribution International License (CC BY). http://creativecommons.org/licenses/by/4.0/

(c) (i) Open Access

\begin{abstract}
We extend our previous analysis and consider the interacting holographic Ricci dark energy (IRDE) model in non-flat universe. We study astrophysical constraints on this model using the recent observations including the type Ia supernovae (SNIa), the baryon acoustic oscillation (BAO), the cosmic microwave background (CMB) anisotropy, and the Hubble parameter. It is shown that the allowed parameter range for the fractional energy density of the curvature is $-\mathbf{0 . 0 0 5} \lesssim \Omega_{\mathrm{k} 0} \lesssim 0.015$ in the presence of the interactions between dark energy and matter. Without the interaction, the flat universe is observationally disfavored in this model.
\end{abstract}

\section{Keywords}

Dark Energy, Ricci, Non-Flat

\section{Introduction}

The current astrophysical observations of the Type Ia supernovae (SNIa) [1] [2], the cosmic microwave background (CMB) [3]-[10] and the large scale structure (LSS) [11] have revealed that the expansion of our universe is accelerated [1] [2]. This indicates that there exists some unknown energy, called dark energy, to realize the accelerated expansion. The simplest interpretation of dark energy is the cosmological constant. However, this model requires an incredible fine-tuning, since the observed cosmological constant is extremely small compared to the fundamental Planck scale $\rho_{\Lambda} \sim 10^{-120} M_{p}^{4}$. Also, this model suffers from the cosmic coincidence problem: why the cosmological constant and matter have comparable energy density today even though their time evolution is so different.

Among various attempts to solve these problems, we focus on the holographic dark energy (HDE) models [12] motivated by the holographic principle of quantum gravity [13]-[16]. Requiring that the total vacuum energy of

${ }^{*}$ Corresponding author. 
a system with size $L$ would not exceed the mass of the black hole of the same size, the dark energy density is proposed as

$$
\rho_{\Lambda}=3 c^{2} M_{p}^{2} L^{-2}
$$

where $c$ is a dimensionless parameter, $M_{P}=1 / \sqrt{8 \pi G}$ is the reduced Planck mass. As for the size $L$, which is regarded as an IR cutoff, various possibilities are discussed in literatures, such as the Hubble parameter $L^{-1}=H$ [12], the future event horizon $L=R_{h}$ [12], the age of our universe $L=T$ [17], and the Ricci scalar curvature $L^{-2}=\mathcal{R}$ [18]. With a reasonable choice of $L$, Equation (1) naturally explains the observed value $\rho_{\Lambda} \sim M_{p}^{2} H_{0}^{2}$ ( $H_{0}$ is the present Hubble parameter). In our previous work [19], we studied the Ricci dark energy (RDE) model with $L^{-2}=\mathcal{R}$ by introducing an interaction between dark energy and matter. It was shown that a nonvanishing interaction rate $Q \propto H \rho_{\Lambda}$ is favored by the observations [19].

In this paper, we extend our previous analysis, and consider the interacting RDE (IRDE) model in non-flat universe. This paper is organized as follows. In Section 2, we describe the generalized IRDE model in non-flat universe, and obtain analytic expressions for cosmic time evolution. In Section 3, we discuss the observational constraints on this model. We summarize our results in Section 4.

\section{The Interacting Ricci Dark Energy Model}

We study the interaction Ricci Dark Energy (IRDE) model in non-flat universe. The Friedmann-RobertsonWalker metric non-flat univrerse is given by

$$
\mathrm{d} s^{2}=\mathrm{d} t^{2}-a^{2}(t)\left(\frac{\mathrm{d} r^{2}}{1-k r^{2}}+r^{2} \mathrm{~d} \theta^{2}+r^{2} \sin ^{2} \theta \mathrm{d} \phi^{2}\right)
$$

where $k=1,0,-1$ for closed, flat, and open geometries. The Friedmann Equation in non-flat univrerse takes the form

$$
H^{2}=\frac{1}{3 M_{p}^{2}}\left(\rho_{\Lambda}+\rho_{m}+\rho_{\gamma}+\rho_{k}\right)
$$

where $\rho_{\Lambda}, \rho_{m}, \rho_{\gamma}$ and $\rho_{k}$ represent energy density of dark energy, matter, radiation and curvature, respectively, and $H=\dot{a} / a$ is the Hubble parameter.

We generalize the energy density of the Ricci dark energy as

$$
\rho_{\Lambda}=3 M_{p}^{2}\left(\frac{\alpha}{2} \partial_{x} H^{2}+\beta H^{2}+\epsilon k \mathrm{e}^{-2 x}\right)
$$

where $\alpha, \beta$ and $\epsilon$ are dimensionless parameters and $x=\ln a$. In the case of $\beta=2 \alpha, \epsilon=\alpha, k=0$, this model is reduced to the ordinary RDE model [19]. Moreover, we introduce a phenomenological interaction between dark energy and matter. The energy densities $\rho_{\Lambda}$ and $\rho_{m}$ obey the following Equations [20]-[40]

$$
\begin{gathered}
\dot{\rho}_{\Lambda}+3 H\left(1+w_{\Lambda}\right) \rho_{\Lambda}=-Q \\
\dot{\rho}_{m}+3 H \rho_{m}=Q
\end{gathered}
$$

We adopt the interaction rate given by

$$
Q=\gamma H \rho_{\Lambda}
$$

where $\gamma$ is a dimensionless parameter [19]. To solve $H$, combining with Equations (4) and (6), the Friedmann Equation (3) is transformed as

$$
\frac{\alpha}{2} \frac{\mathrm{d}^{2} H^{2}}{\mathrm{~d} x^{2}}-\left(1-\frac{3 \alpha}{2}-\frac{\alpha \gamma}{2}-\beta\right) \frac{\mathrm{d} H^{2}}{\mathrm{~d} x}-(3-3 \beta-\beta \gamma) H^{2}-\Omega_{\gamma 0} H_{0}^{2} \mathrm{e}^{-4 x}-\{1-\epsilon(1+\gamma)\} k \mathrm{e}^{-2 x}=0
$$

The solution to Equation (8) is given by 


$$
\frac{H^{2}}{H_{0}^{2}}=A_{+} \mathrm{e}^{\sigma_{+} x}+A_{-} \mathrm{e}^{\sigma_{-} x}+A_{\gamma} \mathrm{e}^{-4 x}+A_{k} \mathrm{e}^{-2 x}
$$

where

$$
\sigma_{ \pm}=\frac{(2-3 \alpha-\alpha \gamma-2 \beta) \pm \sqrt{(2+3 \alpha-2 \beta)^{2}+\alpha \gamma(6 \alpha+\alpha \gamma-4 \beta-4)}}{2 \alpha}
$$

$\Omega_{\gamma 0}=\rho_{\gamma 0} / \rho_{c 0}, \Omega_{k 0}=-k / H_{0}^{2}$ and $\rho_{c 0}=3 M_{p}^{2} H_{0}^{2}$. When $\sigma_{ \pm}$can be imaginary for sufficiently large $\alpha, \beta$ and $\gamma, H^{2}$ has oscillatory behavior [19]. The constants $A_{\gamma}, A_{k}$ and $A_{ \pm}$are obtained as

$$
\begin{gathered}
A_{\gamma}=\frac{\Omega_{\gamma 0}}{(2 \alpha-\beta)(1-\gamma)+1} \\
A_{k}=\frac{1-(1+\gamma) \epsilon}{1+(\alpha-\beta)(1+\gamma)} \Omega_{k 0} \\
A_{ \pm}= \pm \frac{\alpha\left(\sigma_{\mp}+4\right) A_{\gamma}+\alpha\left(\sigma_{\mp}+2\right) A_{k}+2 \Omega_{\Lambda 0}+2 \varepsilon \Omega_{k 0}-\left(\alpha \sigma_{-}+2 \beta\right)}{\alpha\left(\sigma_{+}-\sigma_{-}\right)} .
\end{gathered}
$$

In the case of $\beta=2 \alpha, \epsilon=\alpha$ and $\Omega_{k 0}=0$, Equation (9) reduces to the result obtained in our previous work [19]. Substituting Equation (9) to Equation (4), the Ricci dark energy density is obtained as

$$
\rho_{\Lambda}=\rho_{c 0}\left\{\sum_{i}\left(\frac{\alpha \sigma_{i}}{2}+\beta\right) A_{i} \mathrm{e}^{\sigma_{i} x}-\epsilon \Omega_{k 0} \mathrm{e}^{-2 x}\right\}
$$

where $i \in\{+,-, \gamma, k\}, \sigma_{\gamma}=-4, \sigma_{k}=-2$, Likewise, the matter density is obtained as

$$
\rho_{m}=\rho_{c 0}\left\{\sum_{i}\left(1-\frac{\alpha \sigma_{i}}{2}-\beta\right) A_{i} \mathrm{e}^{\sigma_{i} x}-\Omega_{\gamma 0} \mathrm{e}^{-4 x}-(1-\epsilon) \Omega_{k 0} \mathrm{e}^{-2 x}\right\} .
$$

To derive the Equation of state parameter $w_{\Lambda}$ of the Ricci dark energy, substituting Equation (14) into the following expression:

$$
w_{\Lambda}=-1-\frac{1}{3}\left(\gamma+\frac{1}{\rho_{\Lambda}} \frac{\mathrm{d} \rho_{\Lambda}}{\mathrm{d} x}\right)
$$

\section{Observational Constraints}

In this section, we discuss cosmological constraints on the IRDE model in the non-flat universe $(k \neq 0)$ obtained from SNIa, CMB, BAO and the Hubble parameter observations.

The luminosity distance in the non-flat universe can be written as

$$
d_{L}=\frac{(1+z)}{H_{0} \sqrt{\Omega_{k 0}}} \sinh \left(H_{0} \sqrt{\Omega_{k 0}} \int_{0}^{z} \frac{d z^{\prime}}{H\left(z^{\prime}\right)}\right) .
$$

The SNIa observations measure the distance modulus $\mu$ of a supernova and its redshift $z$. The distance modulus is given by

$$
\mu=5 \log _{10} \frac{d_{L}}{\mathrm{Mpc}} .
$$

We use the Union data set of 580 SNIa [41] [42] to obtain limits on the relevant parameters $\alpha, \gamma$ and $\Omega_{\Lambda 0}$ by minimizing $\chi_{\mathrm{SN}}^{2}[43]-[46]$. 
The CMB shift parameter $R$ is given by

$$
R=\sqrt{\frac{\Omega_{m 0}}{\Omega_{k 0}}} \sinh \left(H_{0} \sqrt{\Omega_{k 0}} \int_{0}^{z_{\mathrm{CMB}}} \frac{\mathrm{d} z}{H(z)}\right)
$$

where $z_{\mathrm{CMB}}=1089$ is the redshift at recombination, and $\Omega_{m 0}=\rho_{m 0} / \rho_{c 0}$ is the matter fraction at present. We use the value $R=1.725 \pm 0.018$ obtained from the WMAP9 data [3]-[10]. The CMB constraints are obtained by minimizing $\chi_{\text {СMB }}^{2}$ [43]-[46]. The shift parameter gives a complementary bound to the SNIa data $(z \lesssim 2)$, since this parameter involves the large redshift behavior $(z \sim 1000)$.

Signatures of the baryon acoustic oscillation (BAO) are provided by the observations of large-scale galaxy clustering. The BAO parameter $A$ is defined by

$$
A=\sqrt{\Omega_{m 0}}\left(\frac{H_{0}}{H\left(z_{\mathrm{BAO}}\right)}\right)^{1 / 3}\left[\frac{1}{Z_{\mathrm{BAO}} \sqrt{\Omega_{k 0}}} \sinh \left(H_{0} \sqrt{\Omega_{k 0}} \int_{0}^{z_{\mathrm{BAO}}} \frac{\mathrm{d} z}{H(z)}\right)\right]^{2 / 3}
$$

where $z_{\mathrm{BAO}}=0.35$. We use the measurement of the BAO peak in the distribution of luminous red galaxies (LRGs) observed in SDSS [11]:

$$
A=0.469\left(\frac{0.95}{0.98}\right)^{-0.35} \pm 0.017
$$

The BAO constraints are obtained by minimizing $\chi_{\mathrm{BAO}}^{2} \quad$ [43]-[46].

The Hubble parameter constraints are given by minimizing

$$
\chi_{H}^{2}=\sum_{i}^{28}\left(\frac{H\left(z_{i}\right)-H_{\mathrm{obs}}\left(z_{i}\right)}{\sigma_{H_{i}}}\right)^{2}
$$

where $\sigma_{H_{i}}$ is the $1 \sigma$ uncertainty of the observational $H(z)$ data [47]-[52].

In Figure 1, we plot the Equation of state parameter $w_{\Lambda}$ for $\Omega_{k 0}=-0.1$ (blue), 0 (red) and 0.1 (green) as a function of the scale factor $a$. The dotted and solid lines are the results for the case without interaction $(\gamma=0)$ and with interaction $(\gamma=0.15)$, respectively. The dark energy parameter is fixed as $\alpha=0.45$. Though the

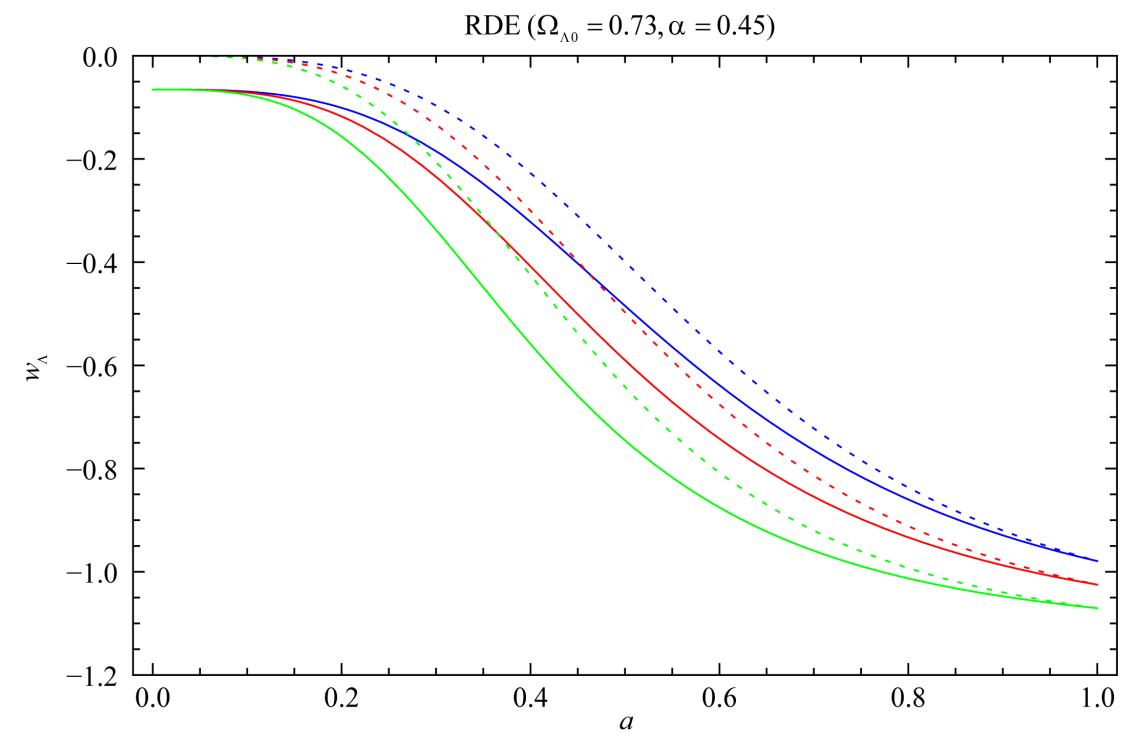

Figure 1. Plot of $w_{\Lambda}$ versus $a$ for $\operatorname{RDE}\left(\Omega_{\Lambda}=0.73, \alpha=0.45, \gamma=0\right)$. The lines for $k=-0.1$ (blue), 0 (red) and 0.1 (green) in the case without interactions (dotted line) and with interaction (solid line). 
value $\Omega_{k 0}= \pm 0.1$ is too large (see Figure 2), we use these values to visualize the effect of the curvature in the figure. In the case of the curvature $k=-0.1$, the value of $w$ is larger than another case, but the condition for accelerated expansion at present $w_{\Lambda}<-1 / 3$ is satisfied for each cases.

In Figure 3, we plot the evolution of the energy density fractions $\Omega$ for radiation (green), matter (red) and dark energy (blue) for $\alpha=0.45$. The dotted and solid lines are the results for the case without interaction $(\gamma=0)$ and with interaction $(\gamma=0.15)$, respectively. The panels (a), (b) and (c) corresponds to $\Omega_{k 0}=0$, -0.1 and 0.1 , respectively. One can see that the effect of the curvature can be important only for $a \sim 1$. The fractions $\Omega_{\Lambda}$ and $\Omega_{m}$ for $\Omega_{k 0}=-0.1$ in the panel (b) $\left(\Omega_{k 0}=0.1\right.$ in the panel (c)) are slightly increased (decreased) around $0.1 \lesssim a \lesssim 1$ compared to the flat case (a). Around radiation-matter equality, the radiation component $\Omega_{\gamma}$ is increased by several percents compared to the corresponding result without interaction (dotted line), while the $\Omega_{m}$ is decreased due to the interaction.

\section{Conclusion}

We have considered the IRDE model in the non-flat universe. We have derived the analytic solutions for the

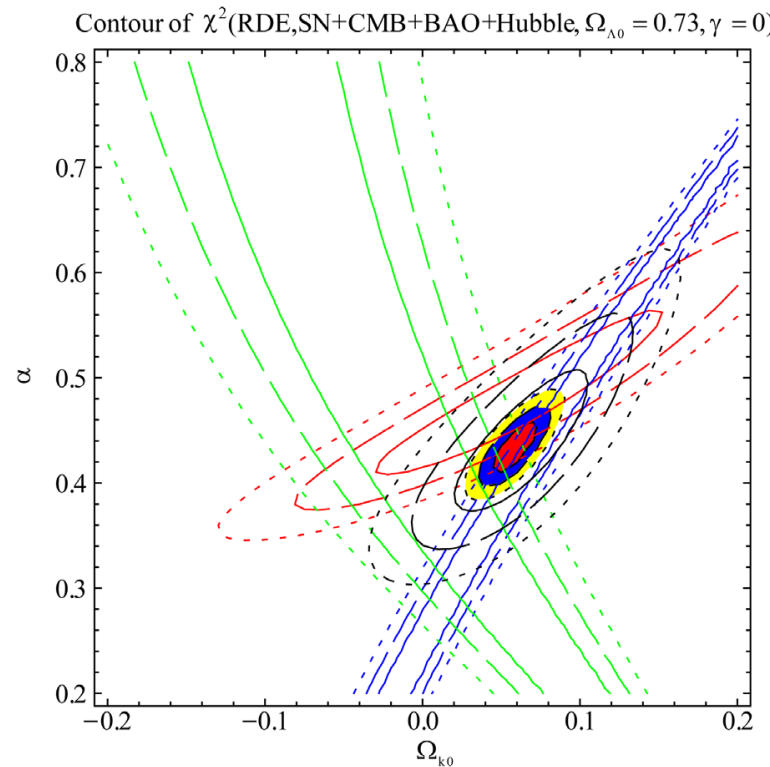

(a)

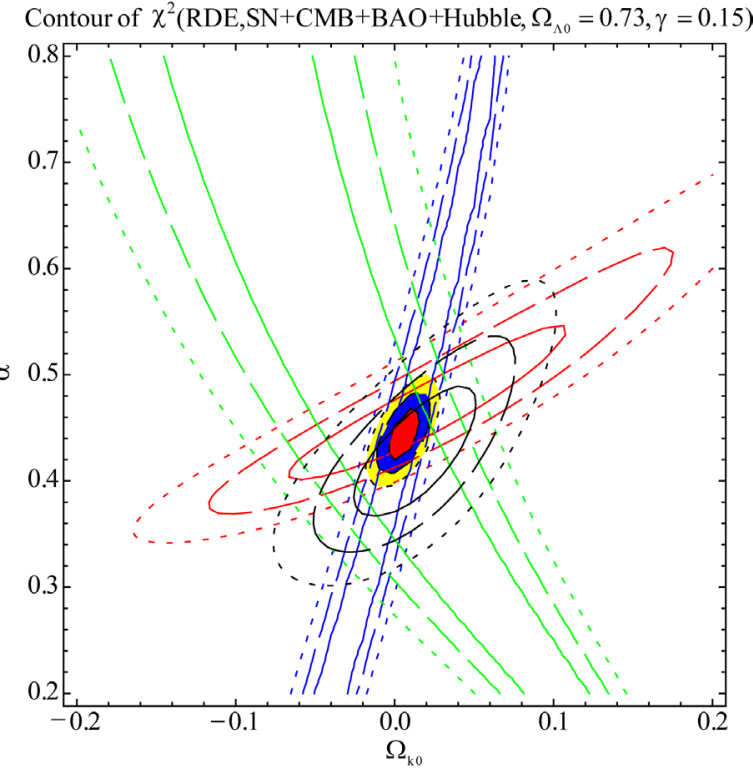

(b)

Figure 2. The probability contours for SNIa (red), CMB (blue), BAO (green) and Hubble (black) observations in the $\left(\alpha, \Omega_{k 0}\right)$-plane in the case without interactions $(\gamma=0)$. The $1 \sigma, 2 \sigma$ and $3 \sigma$ contours are drawn with solid, dashed and dotted lines, respectively. The joined constraints using $\chi^{2}=\chi_{\mathrm{SN}}^{2}+\chi_{\mathrm{CMB}}^{2}+\chi_{\mathrm{BAO}}^{2}+\chi_{H}^{2}$ are shown as shaded contours.

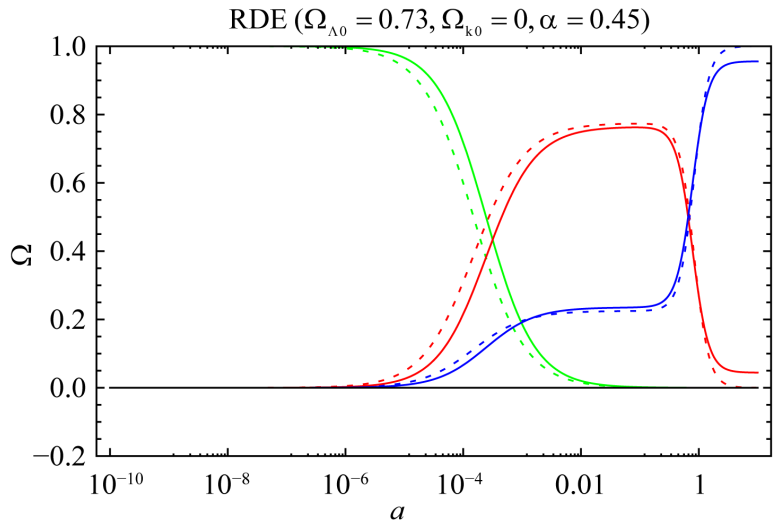

(a)

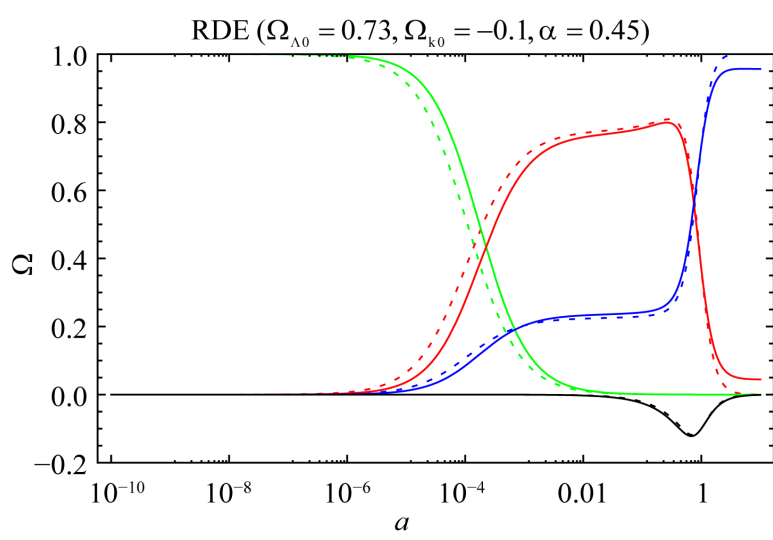

(b) 


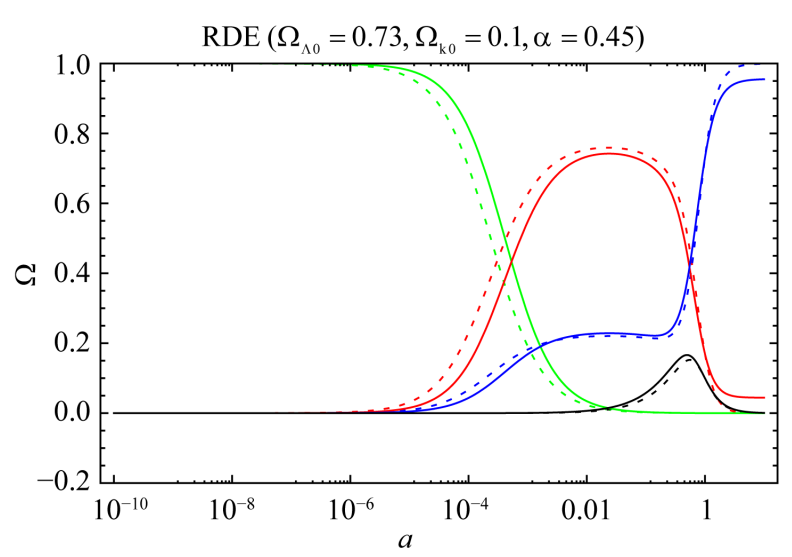

(c)

Figure 3. Plot of $\Omega$ versus $a$ for $\operatorname{RDE}\left(\Omega_{\Lambda}=0.73, \alpha=0.45, \gamma=0.15\right)$. The lines for Radiation (Green), matter (red), dark energy (blue) and curvature (black) in the case without interactions (dotted line) and with interaction (solid line). These figures describe in the case of $\Omega_{k 0}=0$ (a), -0.1 (b) and 0.1 (c).

Hubble parameter (9), the dark energy density (14) and matter energy density (15). We have also studied astrophysical constraints on this model using the recent observations including SNIa, BAO, CMB anisotropy, and the Hubble parameter. We have shown that the allowed parameter range for the fractional energy density of the curvature is $-0.005 \lesssim \Omega_{k 0} \lesssim 0.015$ for $\gamma=0.15$. The best fit values with $1 \sigma$ error are $\Omega_{k 0}=0.006 \pm 0.010$ and $\alpha=0.45 \pm 0.03$ with $\chi_{\min }^{2}=580$. We have shown that the IRDE model with a small curvature is allowed by observational constraints. Without the interaction, the flat universe is observationally disfavored in this model.

\section{Acknowledgements}

We would like to thank T. Nihei for his valuable discussion, helpful advice and reading the manuscript.

\section{References}

[1] Riess, A.G., et al. (1998) The Astronomical Journal, 116, 1009. http://dx.doi.org/10.1086/300499

[2] Perlmutter, S., et al. (1999) The Astronomical Journal, 517, 565. http://dx.doi.org/10.1086/307221

[3] Bennet, C.L., et al. (2003) The Astronomical Journal, 148, 1. http://dx.doi.org/10.1086/377253

[4] Spergel, D.N., et al. (2003) The Astronomical Journal, 148, 175. http://dx.doi.org/10.1086/377226

[5] Spergel, D.N., et al. (2007) The Astronomical Journal, 170, 377. http://dx.doi.org/10.1086/513700

[6] Page, L., et al. (2007) The Astronomical Journal, 170, 335. http://dx.doi.org/10.1086/513699

[7] Hinshaw, G., et al. (2007) The Astronomical Journal, 170, 263. http://dx.doi.org/10.1086/513698

[8] Komatsu, E., et al. (2009) The Astronomical Journal, 180, 330. http://dx.doi.org/10.1088/0067-0049/180/2/330

[9] Komatsu, E., et al. (2011) The Astronomical Journal, 192, 18. http://dx.doi.org/10.1088/0067-0049/192/2/18

[10] Hinshaw, G., Larson, D., Komatsu, E., Spergel, D.N., Bennett, C.L., Dunkley, J., Nolta, M.R., Halpern, M., et al. arXiv:1212.5226 [astro-ph.CO].

[11] Eisenstein, D.J., Zehavi, I., Hogg, D.W., Scoccimarro, R., Blanton, M.R., Nichol, R.C., et al. (2005) Astrophysical Journal, 633, 560-574. http://dx.doi.org/10.1086/466512

[12] Li, M. (2004) Physics Letters B, 603, 1-5. http://dx.doi.org/10.1016/j.physletb.2004.10.014

[13] 't Hooft, G. arXiv:gr-qc/9310026.

[14] Susskind, L. (1995) Journal of Mathematical Physics, 36, 6377. http://dx.doi.org/10.1063/1.531249

[15] Maldacena, J.M. (1998) Advances in Theoretical and Mathematical Physics, 2, 231-252.

[16] Fischler, W. and Susskind, L. (1998) arXiv:hep-th/9806039.

[17] Cai, R.G. (2007) Physics Letters B, 657, 228-231. http://dx.doi.org/10.1016/j.physletb.2007.09.061 
[18] Gao, C., Wu, F., Chen, X. and Shen, Y.G. (2009) Physical Review D, 79, Article ID: 043511. http://dx.doi.org/10.1103/PhysRevD.79.043511

[19] Suwa, M. and Nihei, T. (2010) Physical Review D, 81, Article ID: 023519. http://dx.doi.org/10.1103/PhysRevD.81.023519

[20] Wang, B., Lin, C.Y. and Abdalla, E. (2006) Physics Letters B, 637, 357-361. http://dx.doi.org/10.1016/j.physletb.2006.04.009

[21] Berger, M.S. and Shojaei, H. (2006) Physical Review D, 74, Article ID: 043530. http://dx.doi.org/10.1103/PhysRevD.74.043530

[22] Setare, M.R. (2006) Physics Letters B, 642, 1-4. http://dx.doi.org/10.1016/j.physletb.2006.09.027

[23] Setare, M.R. (2007) [hep-th/0701242].

[24] Wu, Q., Gong, Y., Wang, A. and Alcaniz, J.S. (2008) Physics Letters B, 659, 34-39. http://dx.doi.org/10.1016/j.physletb.2007.10.061

[25] Feng, C., Wang, B., Gong, Y. and Su, R.K. (2007) [arXiv:astro-ph/0706.4033].

[26] Karwan, K. (2008) [arXiv:astro-ph/0801.1755].

[27] Horvat, R. (2004) Physical Review D, 70, Article ID: 087301. http://dx.doi.org/10.1103/PhysRevD.70.087301

[28] Pavon, D. and Zimdahl, W. (2005) Physics Letters B, 628, 206-210. http://dx.doi.org/10.1016/j.physletb.2005.08.134

[29] Guberina, B., Horvat, R. and Nikolic, H. (2007) JCAP, 0701, 012.

[30] Zhang, X. and Wu, F.Q. (2005) Physical Review D, 72, Article ID: 043524. http://dx.doi.org/10.1103/PhysRevD.72.043524

[31] Chang, Z., Wu, F.Q. and Zhang, X. (2006) Physics Letters B, 633, 14-18. http://dx.doi.org/10.1016/j.physletb.2005.10.095

[32] Zhang, X. and Wu, F.Q. (2007) Physical Review D, 76, Article ID: 023502. http://dx.doi.org/10.1103/PhysRevD.76.023502

[33] Zhang, X. (2005) International Journal of Modern Physics D, 14, 1597. http://dx.doi.org/10.1142/S0218271805007243

[34] Setare, M.R., Zhang, J. and Zhang, X. (2007) JCAP, 0703, 007.

[35] Zhang, J., Zhang, X. and Liu, H. (2008) Physics Letters B, 659, 26-33. http://dx.doi.org/10.1016/j.physletb.2007.10.086

[36] Zhang, X. (2007) Physics Letters B, 648, 1-7. http://dx.doi.org/10.1016/j.physletb.2007.02.069

[37] Zhang, X. (2006) Physical Review D, 74, Article ID: 103505. http://dx.doi.org/10.1103/PhysRevD.74.103505

[38] Zhang, J., Zhang, X. and Liu, H. (2007) Physics Letters B, 651, 84-88. http://dx.doi.org/10.1016/j.physletb.2007.06.019

[39] Ma, Y. and Zhang, X. (2008) Physics Letters B, 661, 239-245. http://dx.doi.org/10.1016/j.physletb.2008.02.028

[40] Zhang, J., Zhang, X. and Liu, H. (2007) European Physical Journal C, 52, 693-699. http://dx.doi.org/10.1140/epjc/s10052-007-0408-2

[41] Kowalski, M., Rubin, D., Aldering, G., Agostinho, R.J., Amadon, A., Amanullah, R., et al. (2008) Astrophysical Journal, 686, 749-778. [arXiv:astro-ph/0804.4142] http://dx.doi.org/10.1086/589937

[42] Suzuki, N., Rubin, D., Lidman, C., Aldering, G., Amanullah, R., Barbary, K., et al. (2012) Astrophysical Journal, 746, 85. http://dx.doi.org/10.1088/0004-637X/746/1/85

[43] Nesseris, S. and Perivolaropoulos, L. (2005) Physical Review D, 72, Article ID: 123519. http://dx.doi.org/10.1103/PhysRevD.72.123519

[44] Perivolaropoulos, L. (2005) Physical Review D, 71, Article ID: 063503. http://dx.doi.org/10.1103/PhysRevD.71.063503

[45] Nesseris, S. and Perivolaropoulos, L. (2007) [arXiv:astro-ph/0612653].

[46] Pietro, E.D. and Claeskens, J.F. (2003) Monthly Notices of the Royal Astronomical Society, 341, 1299-1310. http://dx.doi.org/10.1046/j.1365-8711.2003.06508.x

[47] Simon, J., Verde, L. and Jimenez, R. (2005) Physical Review D, 71, Article ID: 123001. http://dx.doi.org/10.1103/PhysRevD.71.123001

[48] Gaztañaga, E., Cabré, A. and Hui, L. (2009) Monthly Notices of the Royal Astronomical Society, 399, 1663-1680. http://dx.doi.org/10.1111/j.1365-2966.2009.15405.x 
[49] Stern, D., et al. (2010) JCAP, 1002, 008.

[50] Moresco, M., et al. (2012) Journal of Cosmology and Astroparticle Physics, 8, 6.

[51] Busca, N.G., Delubac, T., Rich, J., Bailey, S., Font-Ribera, A., Kirkby, D., et al. [arXiv:astro-ph/1211.2616].

[52] Farooq, O., Mania, D. and Ratra, B. [arXiv:astrp-ph/1308.0834]. 